\title{
Self-ordered nanoporous lattice formed by chlorine atoms on $\mathrm{Au}(111)$
}

\author{
V. V. Cherkez, ${ }^{1,2, *}$ V. V. Zheltov, ${ }^{1}$ C. Didiot,${ }^{2, \dagger}$ B. Kierren, ${ }^{2}$ Y. Fagot-Revurat,${ }^{2}$ D. Malterre, ${ }^{2}$ B. V. Andryushechkin,,${ }^{1, \ddagger}$ \\ G. M. Zhidomirov, ${ }^{1,3}$ and K. N. Eltsov ${ }^{1}$ \\ ${ }^{1}$ International Joint Laboratory IMTAS, A. M. Prokhorov General Physics Institute, Russian Academy of Sciences, \\ Vavilov str. 38, 119991 Moscow, Russia \\ ${ }^{2}$ International Joint Laboratory IMTAS, Institut Jean Lamour-UMR CNRS 7198-équipe 102, Departement Physique de la Matière et des \\ Matériaux, B.P 239 Université H. Poincaré-Nancy, 54506 Vandoeuvre les Nancy, France \\ ${ }^{3}$ G. K. Boreskov Institute of Catalysis, Siberian Branch of the Russian Academy of Sciences, Prospect Lavrentieva 5, \\ 630090 Novosibirsk, Russia
}

(Received 8 April 2015; revised manuscript received 11 November 2015; published 28 January 2016)

\begin{abstract}
A self-ordered nanoporous lattice formed by individual chlorine atoms on the $\mathrm{Au}(111)$ surface has been studied with low-temperature scanning tunneling microscopy, low-energy electron diffraction, and density functional theory calculations. We have found out that room-temperature adsorption of 0.09-0.30 monolayers of chlorine on $\mathrm{Au}(111)$ followed by cooling below $110 \mathrm{~K}$ results in the spontaneous formation of a nanoporous quasihexagonal structure with a periodicity of $25-38 \AA$ depending on the initial chlorine coverage. The driving force of the superstructure formation is attributed to the substrate-mediated elastic interaction.
\end{abstract}

DOI: 10.1103/PhysRevB.93.045432

\section{INTRODUCTION}

Adsorption of chlorine on the (111) plane of gold has been investigated both experimentally [1-6] and theoretically [3,5-8]. The first detailed study of $\mathrm{Cl}_{2}$ interaction with the $\mathrm{Au}(111)$ surface in ultrahigh vacuum (UHV) conditions was performed by Spencer and Lambert [1] who detected two different chemical states in thermodesorption spectra. Kastanas and Koel [2] explored adsorption of molecular chlorine onto $\mathrm{Au}(111)$ in a wide temperature range of 100 $400 \mathrm{~K}$ using Auger electron spectroscopy (AES), thermodesorption spectroscopy, and low-energy electron diffraction. They confirmed the presence of two peaks in thermodesorption spectra and reported the ordering of the chlorine overlayer at low temperature and the formation of the dense $(\sqrt{3} \times \sqrt{3}) R 30^{\circ}$ structure corresponding to $\mathrm{Cl}$ coverage $(\theta)$ of 1.33 monolayers (ML) [2]. Gao et al. [3] studied chlorine structures on $\mathrm{Au}(111)$ with scanning tunneling microscopy (STM), low-energy electron diffraction (LEED), and density functional theory (DFT) techniques in the temperature range of $120-750 \mathrm{~K}$. They have established that chlorine adsorption results in the formation of a $(\sqrt{3} \times \sqrt{3}) R 30^{\circ}$ chlorine lattice with one chlorine atom per unit cell at coverage of 0.33 ML. They also performed DFT calculations and found that chlorine atoms occupy fcc positions [3]. This conclusion was confirmed in the LEED study by Zheleva et al. [4].

At coverages exceeding 0.33 ML, the formation of the $\mathrm{AuCl}_{2}$ quasimolecules has been detected in STM [5]. At saturation (0.8 ML), the $\mathrm{AuCl}_{2}$ quasimolecules and chemisorbed chlorine atoms form a complex "honeycomb" structure [3,5].

Initial stage of chlorine adsorption on $\mathrm{Au}(111)(<0.1 \mathrm{ML})$ was investigated with LT-STM in the work by Zheltov et al. [6].

\footnotetext{
*Present address: CEA Saclay, F-91191 Gif sur Yvette Cedex, France.

${ }^{\dagger}$ Current address: Université de Fribourg, Department of Physics, Fribourg, Switzerland.

${ }^{\ddagger}$ andrush@kapella.gpi.ru
}

It was surprisingly found that chlorine can form chains, with atoms alternatively occupying fcc and bridge positions. The interatomic distances within the chains $(3.8 \AA)$ were found to be lower than those $(5.0 \AA)$ in the $(\sqrt{3} \times \sqrt{3}) R 30^{\circ}$ structure observed at $0.33 \mathrm{ML}[3,5]$. This structural paradox was solved using DFT calculations that show an important role of the short-range indirect elastic interactions via substrate lattice distortion. However, further development of chlorine structures from single chains to a complete $(\sqrt{3} \times \sqrt{3}) R 30^{\circ}$ monolayer remains unexplored so far.

In this paper, we present the results of our systematic experimental and theoretical study of chlorine adsorption on $\mathrm{Au}(111)$ for submonolayer coverages $(0.01<\theta<0.33 \mathrm{ML})$. We have found that adsorption of 0.09-0.30 ML of chlorine on the clean $\mathrm{Au}(111)$ surface at room temperature and subsequent cooling below $110 \mathrm{~K}$ leads to the formation of the self-ordered quasihexagonal nanoporous structure formed by individual chlorine atoms. The period of the superstructure was found to be in the range of 25-38 $\AA$ depending on the chlorine coverage. We have examined all steps of the structure formation and its transformation into the $(\sqrt{3} \times \sqrt{3}) R 30^{\circ}$ structure at $0.33 \mathrm{ML}$. The driving force for the nanoporous lattice formation is attributed to the substrate-mediated longrange elastic interaction.

\section{EXPERIMENTAL AND COMPUTATIONAL DETAILS}

The experiments were carried out in an ultrahigh vacuum setup equipped with a chamber for the preparation and characterization of metallic surfaces and an STM chamber with Omicron low-temperature (LT) STM. The Au(111) sample was cleaned by several cycles of $\mathrm{Ar}^{+}$sputtering $(1 \mathrm{keV})$ and annealing $(800 \mathrm{~K})$ and characterized by STM, LEED, and AES. Chlorine gas $\left(\mathrm{Cl}_{2}\right)$ was introduced via a piezoelectric leak valve at a typical pressure of $10^{-10}-10^{-9} \mathrm{mbar}$. Exposure of $0.1-1$ min resulted in a chlorine coverage ranging from 0.001 up to $0.33 \mathrm{ML}$ as measured by STM. The sample was exposed at room temperature $(300 \mathrm{~K})$ and further cooled down to 5 , 50, or $77 \mathrm{~K}$ inside STM. WSXM software [9] was used for the analysis 

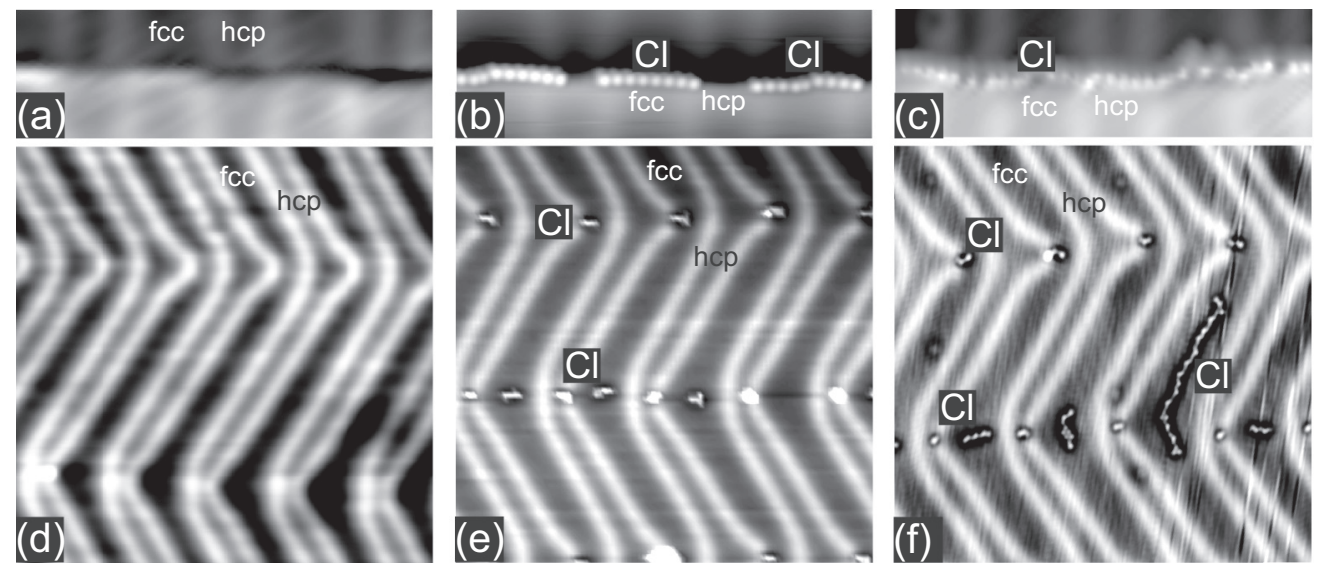

FIG. 1. STM $(5 \mathrm{~K})$ images of the $\operatorname{Au}(111)-(22 \times \sqrt{3})$ surface before and after adsorption of small dose of chlorine $(\theta<0.01 \mathrm{ML})$. (a) Atomic step of the clean surface $\left(180 \times 55 \AA^{2}, \mathrm{U}_{s}=-100 \mathrm{mV}, \mathrm{I}_{t}=0.5 \mathrm{nA}\right)$. (b) Chlorine atoms adsorbed on the step edge in fcc domains $\left(180 \times 55 \AA^{2}, \mathrm{U}_{s}=-100 \mathrm{mV}, \mathrm{I}_{t}=0.5 \mathrm{nA}\right)$. (c) Fully decorated atomic step $\left(180 \times 55 \AA^{2}, \mathrm{U}_{s}=-250 \mathrm{mV}, \mathrm{I}_{t}=1 \mathrm{nA}\right)$. (d) Atomic terrace with the $\mathrm{Au}(111)-(22 \times \sqrt{3})$ reconstruction $\left(360 \times 360 \AA^{2}, \mathrm{U}_{s}=-100 \mathrm{mV}, \mathrm{I}_{t}=0.5 \mathrm{nA}\right)$. (e) Chlorine atoms adsorbed in kinks of the surface reconstruction $\left(360 \times 360 \AA^{2}, \mathrm{U}_{s}=-1 \mathrm{~V}, \mathrm{I}_{t}=1.5 \mathrm{nA}\right)$. (f) Nucleation and growth of single atomic chains of chlorine in fcc domains $\left(360 \times 360 \AA^{2}, \mathrm{U}_{s}=-1 \mathrm{~V}, \mathrm{I}_{t}=1.5 \mathrm{nA}\right)$. Chlorine dose in (c) and (f) is larger than in (b) and (e).

of STM data. LEED measurements were performed in the same setup at sample temperatures in the range of 50-300 K.

The DFT calculations were performed with the periodic plane-wave basis set code VASP 5.3 [10-13] and PBE exchange-correlation functional [14]. All results have been obtained with projector-augmented-wave [15] potentials using a $350-\mathrm{eV}$ plane-wave cutoff. The $\mathrm{Au}(111)-(1 \times 1)$ substrate was modeled by a two-layer slab of a $12 \times 12$ unit cell. We used a Monkhorst-Pack [16] $k$-point grid of $2 \times 2 \times 1$ and $15-\AA$ vacuum region between the slabs.

\section{RESULTS AND DISCUSSION}

A. Initial stage of chlorine adsorption, formation of nanoporous structure, and lifting of herringbone reconstruction on $\mathrm{Au}(111)$

Clean $\mathrm{Au}(111)$ surface is known to be reconstructed $[17,18]$. In this reconstruction, the density of gold atoms in the upper layer exceeds by $4 \%$ the density of atoms in the (111) plane in the bulk. As a result, the $(22 \times \sqrt{ } 3)$ structure is formed in which surface Au atoms occupy fcc, hcp, and bridge sites. Typical STM images of the clean Au(111) surface are shown in Figs. 1(a) and 1(d). The bright stripes correspond to the regions with increased density of atoms (domain walls or soliton lines) separating fcc and hep domains. Periodicity of the structure in the direction perpendicular to domain walls is about $65 \AA$, with fcc domains being broader (40 $\mathrm{A}$, as measured from our data) than hcp domains ( $25 \AA)$. The zigzag shape of domains is explained by secondary reconstruction that removes excessive elastic stress from the surface [18]. The length of domains between elbows is about $220 \AA$.

Adsorption of a small amount of chlorine $(\theta<0.01 \mathrm{ML})$ leads first to the occupation of positions on the step edges belonging to fcc domains on the terrace [Fig. 1(b)]. In parallel, chlorine adsorbs in fcc domains near defects in the gold lattice situated in the elbows of the $\mathrm{Au}(111)$ reconstruction. Such behavior is not surprising since adsorption activity of these sites on the $\mathrm{Au}(111)-(22 \times \sqrt{3})$ surface has been reported in many adsorption studies [19-21]. After full occupation of these sites, chlorine adsorbs in the rest of the sites on the step edges [within hcp domains, see Fig. 1(c)]. These experimental observations are in line with DFT calculations by Baker et al. [8] predicting the increase of the adsorption energy of chlorine near defects on the Au(111) surface.

At higher coverage, chlorine atoms start to fill fcc domains between kinks of the reconstruction [see Figs. 1(f) and 2]. At the beginning, they form linear chains in the centers of fcc domains [Fig. 2(a)]. Two different atomic configurations have been found: the hexagonal atomic packing labeled as A and the zigzag arrangement of atoms labeled as B [6]. In configuration $\mathrm{A}$, all chlorine atoms occupy equivalent fcc adsorption sites with nearest-neighbor distances of $5.0 \AA$ [like in the $(\sqrt{3} \times \sqrt{3}) R 30^{\circ}$ structure formed at $\left.\frac{1}{3} \mathrm{ML}\right]$. Atoms in configuration $\mathrm{B}$ are separated by the unusually small distance of $3.8 \AA$. Two different adsorption sites have been detected for configuration B: bridge and fcc. Moreover, chlorine atoms situated in bridge adsorption sites appear to be brighter in STM images than their darker neighbors occupying fcc positions [Fig. 2(b)]. Such unusual adsorption of chlorine atoms in alternating bridge-fcc sites has been explained by substratemediated elastic attraction between adsorbed atoms [6]. The coexistence of both structures has been explained by almost the same value of adsorption energy [6].

Strictly speaking, we can not state definitely that chlorine adsorbs exclusively in the center of fcc domains since fcc domains containing chlorine chains appear to be broadened by a factor of 1.5 in comparison to those of the clean $\mathrm{Au}(111)-(22 \times \sqrt{3})$ surface (see Fig. 2). Therefore, the appearance of chains may already be seen as a first step towards lifting the herringbone reconstruction. Nevertheless, we can refer to analogy with results of theoretical works on electronegative adsorbates (fluorine, sulfur) on the $\mathrm{Au}(111)-(22 \times \sqrt{3})$ surface $[22,23]$. According to DFT calculations, the minimum of adsorption energy for fluorine [22] and sulfur [23] corresponds to the position in the center of fcc domain. 


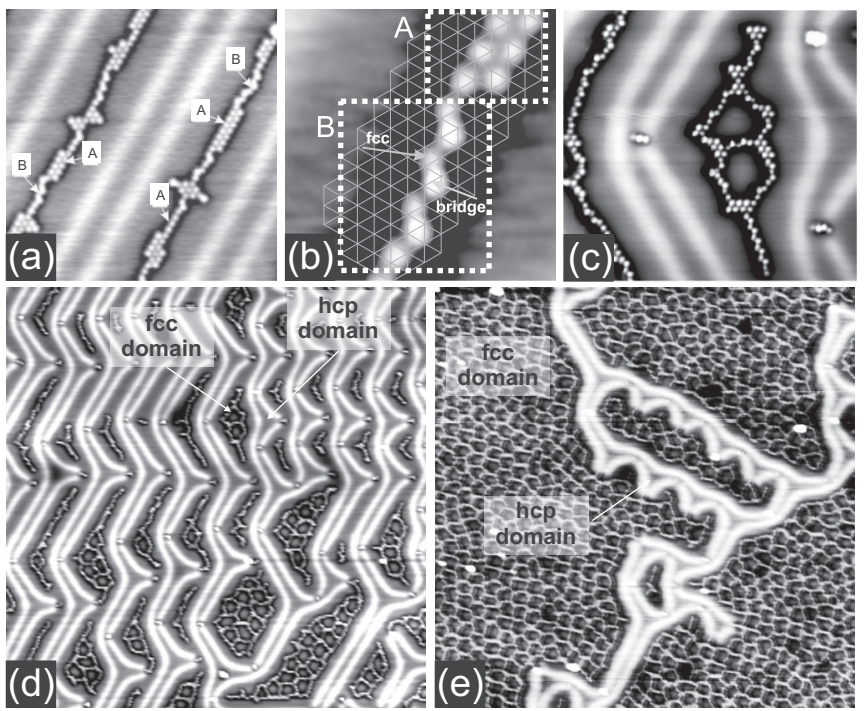

FIG. 2. (a) STM image $\left(230 \times 230 \AA^{2}, \mathrm{U}_{s}=-1 \mathrm{~V}, \mathrm{I}_{t}=0.5 \mathrm{nA}\right.$, $5 \mathrm{~K}$ ) of linear chlorine chains growing in fcc domains. Two types of stacking of chlorine atoms: fcc-fcc (A) and fcc-bridge (B) are indicated. (b) STM image of the linear structure from (a) shown with high magnification. The hexagonal net indicating the $\mathrm{Au}(111)-(1 \times 1)$ lattice is superimposed onto the STM image. (c) STM image $(230 \times$ $\left.230 \AA^{2}, \mathrm{U}_{s}=-1 \mathrm{~V}, \mathrm{I}_{t}=0.5 \mathrm{nA}, 5 \mathrm{~K}\right)$ showing the nucleation of nanopores. (d), (e) STM images $\left(1000 \times 1000 \AA^{2}, \mathrm{U}_{s}=-1 \mathrm{~V}, \mathrm{I}_{t}=\right.$ $0.5 \mathrm{nA}, 5 \mathrm{~K})$ demonstrating lifting of the herringbone reconstruction in the course of chlorine adsorption. (d) $\theta \approx 0.01 \mathrm{ML}$. (e) $\theta \approx$ $0.08 \mathrm{ML}$

As chlorine coverage grows, the length of atomic chains increases up to $\approx 200 \AA$ being limited only by the length of fcc domains of the herringbone reconstruction. Chlorine chains can also bend and form closed rings [see Fig. 2(c)]. First rings with average size about $30-50 \AA$ usually appear in the elbows of the herringbone reconstruction. Further increase of chlorine coverage leads to the formation of several bonded rings (nucleus of the porous structure) inside fcc domains [Fig. 2(d)].

It should be noted that surface coverage is very inhomogeneous at this stage of adsorption. Large regions of several tens of nanometers are filled with the nanoporous structure and correspond to a local coverage $\theta \approx 0.08-0.09 \mathrm{ML}$, whereas the rest of the surface is covered by the residue of the initial reconstruction covered with less than 0.01 ML of chlorine [see Fig. 2(e)]. Surface reconstruction in the latter regions is disturbed and domain walls have unusual shape. At coverage of $\theta \approx 0.12 \mathrm{ML}$, the reconstruction is completely lifted and the surface is completely covered by nanoporous superstructure.

The effect of adsorbates on the reconstruction was studied previously in a number of STM works [24-28]. It was found out that electronegative adsorbates $\left(\mathrm{S}, \mathrm{O}, \mathrm{ClO}_{4}^{-}\right)$induce compressive stress compensating for the tensile stress on the clean $\mathrm{Au}(111)$ surface. In particular, adsorption of sulfur was found to lift reconstruction completely [25,26]. Adsorption of oxygen resulted in disappearance of elbows of the herringbone reconstruction persisting the soliton walls [25]. In electrochemical environment, adsorption of $\mathrm{ClO}_{4}^{-}$ions on the $\mathrm{Au}(111)-(22 \times \sqrt{3})$ surface also resulted in lifting of the reconstruction [24]. In contrast, alkali metals induce the tensile stress. For instance, in Ref. [28] it was demonstrated that adsorption of sodium on the reconstructed $\mathrm{Au}(111)$ surface resulted only in modification of the soliton walls and higher corrugation in STM images.

\section{B. Evolution of nanoporous structure with chlorine coverage $(0.09<\theta<0.33 \mathrm{ML})$}

In this section, we consider the evolution of the local structure of the nanoporous lattice with chlorine coverage. The top row in Fig. 3 shows LT-STM images of the $\mathrm{Au}(111)$ surface
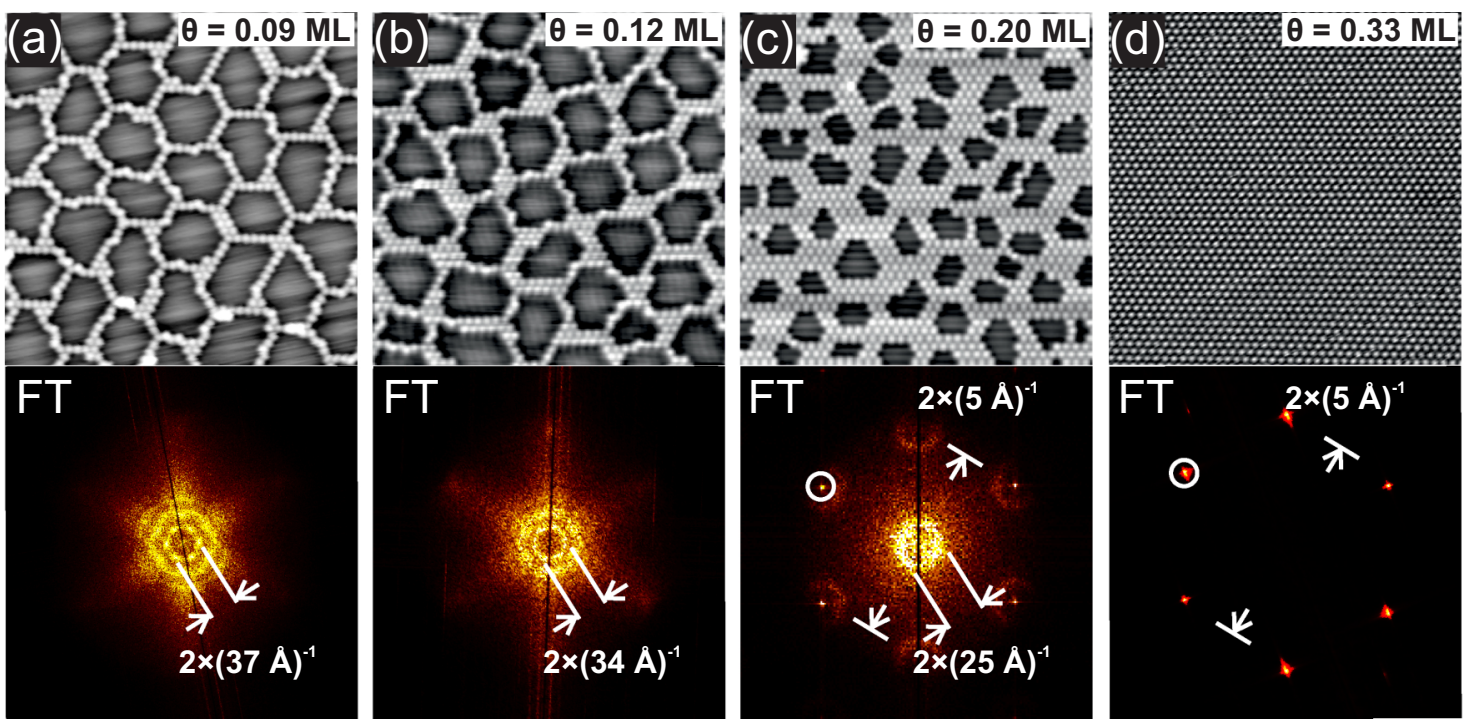

FIG. 3. STM images $\left(200 \times 200 \AA^{2}, 5 \mathrm{~K}\right)$ showing evolution of the nanoporous lattice with chlorine coverage (top row) and corresponding Fourier transforms (FT) (bottom row). (a) $\theta \approx 0.09 \mathrm{ML}$; (b) $0.12 \mathrm{ML}$; (c) $0.2 \mathrm{ML}$; (d) $0.33 \mathrm{ML}$. Positions of the $(\sqrt{3} \times \sqrt{3}) R 30^{\circ}$ spots in the reciprocal space are shown by white circles. Scanning parameters: (a) $\mathrm{U}_{s}=-1.0 \mathrm{~V}, \mathrm{I}_{t}=0.5 \mathrm{nA}$; (b) $\mathrm{U}_{s}=-1.0 \mathrm{~V}, \mathrm{I}_{t}=1.0 \mathrm{nA}$; (c) $\mathrm{U}_{s}=-0.5 \mathrm{~V}, \mathrm{I}_{t}=1.5 \mathrm{nA} ;(\mathrm{d}) \mathrm{U}_{s}=-1.0 \mathrm{~V}, \mathrm{I}_{t}=1.0 \mathrm{nA}$. 


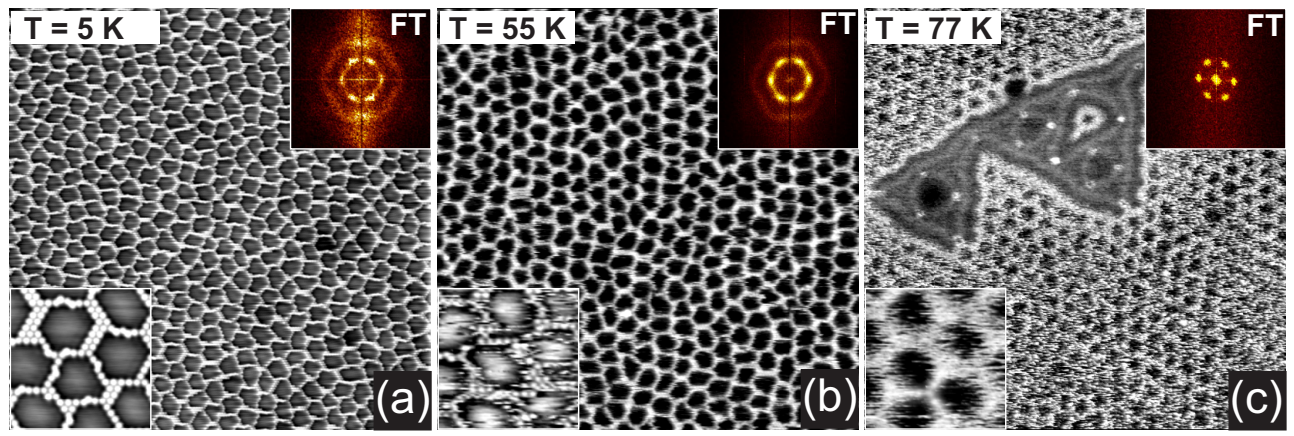

FIG. 4. Large-scale STM images $\left(800 \times 800 \AA^{2}, \mathrm{U}_{s}=2.0 \mathrm{~V}, \mathrm{I}_{t}=0.5 \mathrm{nA}\right)$ of the nanoporous structure $(\theta=0.11 \mathrm{ML})$ on the Au(111) surface at $5 \mathrm{~K}(\mathrm{a}), 55 \mathrm{~K}(\mathrm{~b})$, and $77 \mathrm{~K}(\mathrm{c})$. Corresponding FT-STM images are shown in the insets to each image. The magnified fragments of the STM images $\left(90 \times 90 \AA^{2}\right)$ are shown in bottom-left corners.

with chlorine superstructures corresponding to chlorine coverages in the range of $0.09-0.33 \mathrm{ML}$, as estimated from STM images. Self-ordering and approximately hexagonal symmetry of the structure can be evidenced by the Fourier transform of the STM images (FT-STM) presented in the bottom row in Fig. 3 .

A self-ordered nanoporous structure built from singleatomic chains is shown in Fig. 3(a). It corresponds to chlorine coverage of $\approx 0.09 \mathrm{ML}$. This is the minimal local surface coverage to observe nanoporous superstructure. Chlorine atoms inside walls can occupy fcc or bridge sites as observed for single-atomic chains described above. A clear sixfold pattern with second- and third-order spots is visible in the FT-STM image. The periodicity of the structure estimated from FT is equal to $37 \mathrm{~A}$. For slightly higher chlorine coverage, the atomic structure of the pore walls changes [Fig. 3(b)]: they become thicker, with chlorine atoms occupying predominantly fcc adsorption sites in the commensurate $(\sqrt{3} \times \sqrt{3}) R 30^{\circ}$ structure. The superstructure becomes less ordered, however, a diffuse hexagon still can be seen in the FT-STM image. At the same time, the period of the superstructure decreases down to $34 \AA$. Further increase of chlorine coverage leads to a loss of the ordering of pores [Fig. 3(c)]. As the thickness of chlorine walls around pores gradually increases, the FT-STM image shows appearance of additional spots corresponding to the $(\sqrt{3} \times \sqrt{3}) R 30^{\circ}$ periodicity [Fig. 3(c)]. Comparing the position of spots for the nanoporous and the $(\sqrt{3} \times \sqrt{3}) R 30^{\circ}$ structures shows that the rows formed by the pores in the superstructure are aligned along the $\langle 110\rangle$ direction of close-packed atomic rows of the $\mathrm{Au}(111)-(1 \times 1)$ substrate. At $\theta=0.33 \mathrm{ML}$, all pores disappear leaving a complete commensurate $(\sqrt{3} \times \sqrt{3}) R 30^{\circ}$ structure on the surface.

It is noteworthy that partial desorption of chlorine from the $(\sqrt{3} \times \sqrt{3}) R 30^{\circ}$ structure by heating to a certain temperature in the range of $450-600 \mathrm{~K}$ and subsequent cooling to $5 \mathrm{~K}$ results in appearance of the same set of nanoporous structures but in the reverse order. In other words, chlorine structures are fully reversible in relation to adsorption/desorption processes and correspond to chlorine coverage.

\section{Thermal stability of the nanoporous structure}

Figure 4 shows STM images of the nanoporous lattice $(\theta=0.11 \mathrm{ML})$ acquired at three different temperatures: 5, 55, and $77 \mathrm{~K}$. The mobility of chlorine atoms seems to increase very fast with the temperature. At $55 \mathrm{~K}$, it was hard to get atomic resolution, although the superstructure still exists as can be seen in Fig. 4. At higher temperature $(77 \mathrm{~K})$, we failed to get STM images with atomic resolution, which can be explained by fluctuations in atomic positions of chlorine atoms. Nevertheless, even at this elevated temperature the rings and the skeleton of the nanoporous structure still survive as one can see directly from the STM image and its FT [see Fig. 4(c)]. Only small parts of the superstructure are destroyed, but we can not rule out that some of mobility observed is due to the influence of the STM tip.

To explore higher temperatures not accessible with our STM and to see the disappearance of the superstructure, we performed LEED measurements (see Fig. 5) for the same chlorine coverage. At $47 \mathrm{~K}$ (the lowest temperature available for LEED measurements in our setup), we observed sharp spots from the $\mathrm{Au}(111)-(1 \times 1)$ substrate. There is an additional set of spots that are arranged in small hexagons around each spot of the substrate. These additional spots come from the nanoporous superlattice, which according to our STM data still exists on the surface at this temperature. The reciprocal-space
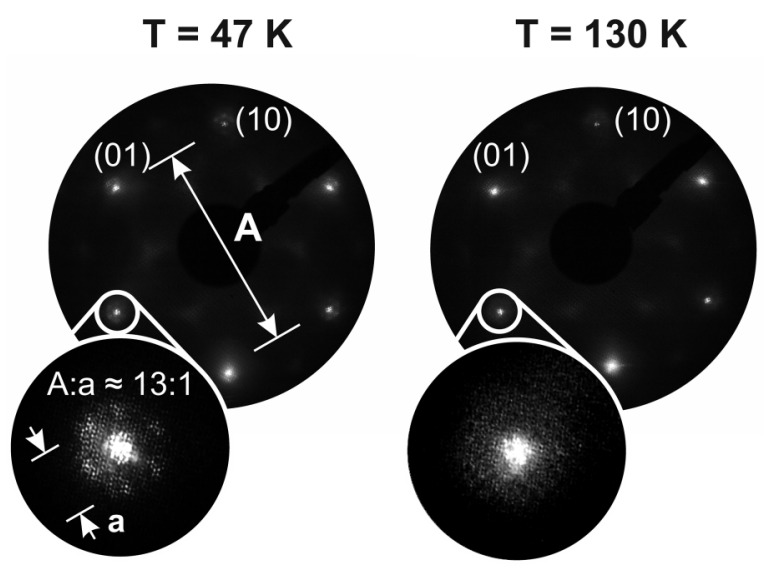

FIG. 5. LEED images $\left(E_{0}=145 \mathrm{eV}\right)$ of the chlorinated $\mathrm{Au}(111)$ surface $(\theta=0.11 \mathrm{ML})$ at substrate temperatures of 47 and $130 \mathrm{~K}$. First-order spots from the substrate are clearly seen. For the LEED pattern taken at $47 \mathrm{~K}$, a high magnification of the area around main spots (shown in the insets) reveals a small hexagon corresponding to the nanoporous superstructure. These spots disappear at $120 \mathrm{~K}$. 
orientation of the substrate and superlattice spots confirms the $\langle 110\rangle$ direction of the rows formed by the pores in the structure. The distance between superstructure spots is roughly 13 times smaller than the distance between substrate spots. This corresponds to the superstructure periodicity of $37.5 \AA$ and is in an agreement with STM measurements for $\theta=0.10-0.11 \mathrm{ML}$. When the temperature of the surface is increased, the spots from chlorine nanoporous structure become more and more diffuse and at temperatures above $120 \mathrm{~K}$, only a diffuse halo is observed around surface spots (Fig. 5). From these LEED experiments, the temperature of the superstructure disordering was estimated to be in the range of $100-120 \mathrm{~K}$. This order-disorder transition is perfectly reversible. Therefore, by cycling of the sample temperature from room temperature down to $5 \mathrm{~K}$, we always restore the nanoporous lattice with the same periodicity at low temperature.

Unfortunately, we have not got enough experimental data to plot the temperature of the order-disorder transition as a function of chlorine coverage. However, from our point of view, the transition temperature should decrease with chlorine coverage increase since the ordering of the nanoporous structure at $0.09 \mathrm{ML}$ is better than at 0.20 ML (see Fig. 3).

\section{Origin of the ordering}

To understand the preferable formation of porous structure at low chlorine coverage on $\mathrm{Au}(111)$, we performed model DFT calculations. The application of DFT calculations to study indirect interactions at surfaces (both electronic and elastic) is quite common [6,29-31]. Distinguishing between electronic and elastic contributions can be done by comparison adsorption/interaction energies obtained in two calculations with fixed and fully relaxed geometries of the substrate [6,29-31]. Using this approach, the elastic part of the indirect interactions was revealed at short adsorbate distances in a number of systems: $\mathrm{Al} / \mathrm{Al}(111), \mathrm{Cu} / \mathrm{Cu}(111), \mathrm{Ag} / \mathrm{Ag}(111)$ [29-31]. In all these systems, however, indirect electronic interactions were found to be the dominant factor in the formation of surface structures. The $\mathrm{Cl} / \mathrm{Au}(111)$ system [6], on the contrary, was found to be an interesting example of the major role of the indirect elastic interactions at short distances $(<5 \AA)$ responsible for the formation of single-atomic chlorine chains with a nearest-neighbor distance of $3.8 \AA$.

We have used a large unit cell $12 \times 12$ with a period of $\approx 35 \AA$ that roughly corresponds to the structure shown in Fig. 3(a). Since the number of atoms in the calculations is limited, the substrate was modeled by a two-layer slab. The lower gold layer was fixed, while the upper layer was allowed to relax. Three model systems have been examined. In the first structure shown in Fig. 6(a), chlorine atoms were placed in fcc sites forming a nanoporous structure. In the second case, all chlorine atoms occupying fcc positions have been collected in a single $(\sqrt{3} \times \sqrt{3}) R 30^{\circ}$ island. In the third model shown in Fig. 6(c), chlorine atoms were spread out more or less uniformly in the unit cell.

As follows from Fig. 6, the nanoporous structure appears to be favorable in the case of calculations with full relaxation of the upper gold layer. The energy gain over the compact structure is equal to $50 \mathrm{meV}$. This value is small, but we

\section{$E_{\text {ads }}, e V / a t o m$ relaxed fixed}

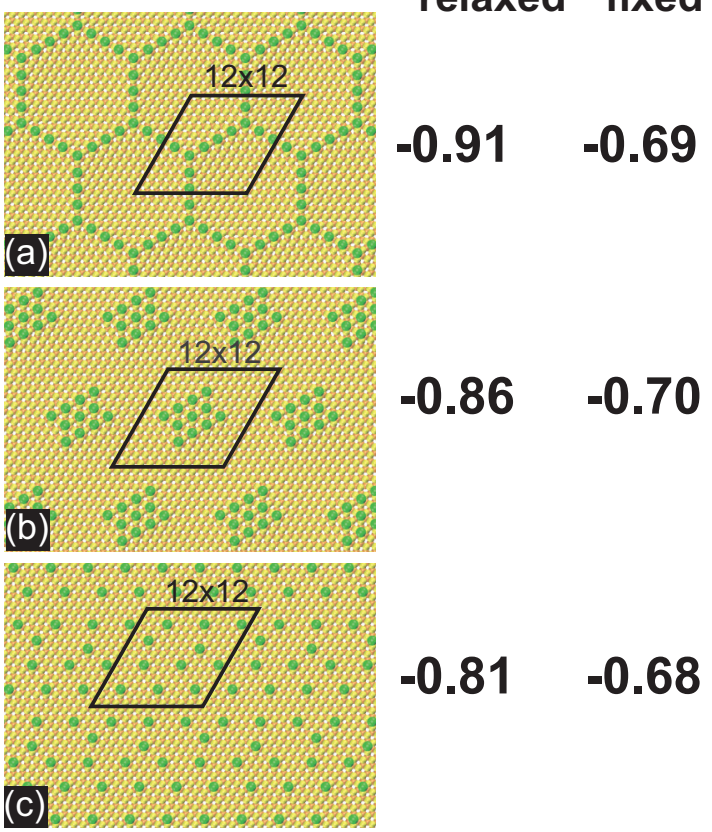

FIG. 6. DFT calculations of adsorption energy for three structures: (a) nanoporous, (b) compact, and (c) disordered. All structures contain equal number of chlorine atoms in $12 \times 12$ unit cell.

consider it reliable since it reflects the difference between two similar systems, where systematic errors of the DFT calculations should cancel out [32]. The errors in this case are known to be rather small $(<10 \mathrm{meV})$. In particular, the difference between hcp and bridge positions about 10 $12 \mathrm{meV}$ was considered to be reliable in the $\mathrm{Cl} / \mathrm{Au}(111)$ system $[3,6]$.

Calculations performed with a fixed geometry of the substrate show no preference of the porous structure. This result indicates the important role of elastic interactions in the formation of the porous structure. Of course, in our calculations, we have considered only three idealized structures. In the real world, chlorine atoms also tend to form zigzag chains with atoms adsorbed in fcc-bridge positions (see Figs. 2 and 3 and Ref. [6]). The local fcc-bridge configuration is formed due to a strong local distortion of the gold lattice [6]. In the present case, we report that long-range elastic interactions (mediated by the substrate lattice distortion) can be responsible for the self-ordering in the chlorine layer.

Figure 7(a) shows the DFT-optimized arrangement of atoms in the upper gold layer corresponding to the model from Fig. 6(a). The clearly visible hexagon with increased interatomic distances indicates stress redistribution in the system. The plot in Fig. 7(b) shows Au-Au distances measured along the straight line marked in Fig. 7(a) as A-A versus number of the $\mathrm{Au}$-Au pair. We see that chlorine atoms induce a significant increase of the interatomic distances up to $3.7 \AA$ $(\approx+26 \%$ with respect to $2.94 \AA$ in the undisturbed lattice) in the sites of adsorption. In the next pair, the distances $(2.82 \AA)$ appear to be smaller than in the undisturbed lattice $(2.94 \AA)$. According to the plot, as we approach the center of the pore, the 

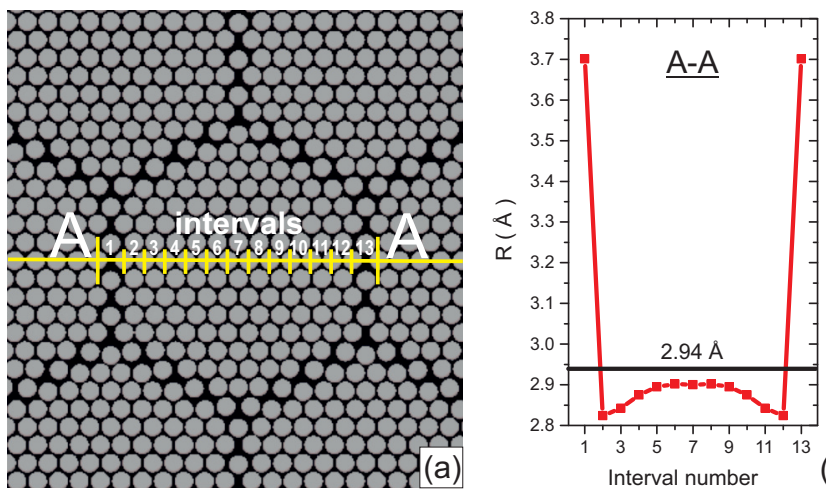

FIG. 7. (a) Relaxation of the upper gold layer corresponding to the model of the porous superstructure from Fig. 6(a). Gold atoms are shown in gray. (b) Plot showing the distribution of $\mathrm{Au}-\mathrm{Au}$ interatomic distances in the cross section A-A from (a). Horizontal black line indicates the value of the Au-Au distance in the DFT optimized $\mathrm{Au}(111)-(1 \times 1)$ lattice.

interatomic distances increase up to $2.9 \AA$. It is worth noting that even in the center of the pore, the Au-Au distances remain shorter than in the undisturbed $(1 \times 1)$ lattice $(2.94 \AA)$. The character of the surface stress is compressive under chlorine atoms and tensile in the rest of the pore. Thus, adsorption of small amount of chlorine $(\theta \approx 0.1 \mathrm{ML})$ gives rise to the formation of the self-consistent periodic stress field in the upper layer of the substrate.

This stress field could be modified by the structural defects: dislocations or steps. Figures 8(a) and 8(b) show the influence of the dislocation on the nanoporous lattice. This type of defect can be created by subsurface implantation of noble gas atoms. For this purpose, we used a routine procedure of the sample preparation in which the temperature of annealing (necessary to remove argon implantations) was reduced from $800 \mathrm{~K}$ down to $600 \mathrm{~K}$. In the large-scale STM images, argon-induced defects perturbing the herringbone reconstruction around them are clearly seen [Fig. 8(a)]. The inset in Fig. 8(a) shows the defect with higher magnification. Analysis of the STM image reveals a step segment aligned parallel to the $\langle 110\rangle$ direction with a height $\approx \frac{1}{3}$ of a full step height. We explain this by the presence of an edge dislocation. The intrinsic stacking fault likely extends to the lower left, visible as the brighter ribbon in STM. Note that similar STM images of the edge dislocations on the $\mathrm{Au}(111)$ surface were published in the work by Engbæk et al. [33]. Thus, at our experimental conditions, implantation of argon atoms in subsurface of $\mathrm{Au}(111)$ gives rise to the formation of edge dislocations. The basic mechanism could be similar to the case described in the work by Micheley and Comsa [34], in which the formation of hexagonal adatom islands on the $\operatorname{Pt}(111)$ surface after ion bombardment was explained by the formation and gliding of the dislocation loops towards the surface.

We have found that the nanoporous lattice of chlorine is strongly distorted around the dislocation [Fig. 8(b)]. Chlorine atoms decorate step segment forming straight lines in $\langle 110\rangle$ directions. In the vicinity of the step segment, the pores have larger average size and are elongated towards the step segment. The superstructure distortion could be explained by a strong local stress on the $\mathrm{Au}(111)$ surface induced by the presence of edge dislocation.

Also note that the STM image in Fig. 8(b) contains a bright object we relate to on-surface contaminations. We see that such type of defect does not disturb the superstructure. This observation is in line with our previous reasoning since onsurface defect does not produce any appreciable distortions of the gold lattice.

Atomic steps are another type of surface defect that can produce strong elastic distortion on the surface [35]. On the vicinal surface, steps are separated by narrow terraces of a regular width. As the result of elastic interaction between steps, the terraces appear to be strongly perturbed compared to the corresponding low-index plane [36,37]. We have adsorbed chlorine on the $\mathrm{Au}(232321)$ surface that consists of closepacked $\{111\}$ terraces with average width of $57 \AA$ and separated by $\langle 111\rangle$-like monoatomic steps [38]. On each terrace, the $(22 \times \sqrt{3})$ reconstruction develops with fcc-hcp domains running perpendicular to the steps. Figure 8(c) shows the structure of $0.06 \mathrm{ML}$ chlorine layer on this surface. Although the width of terraces is sufficient to accommodate pores with single-atom walls and the size of $30-40 \AA$ [as in the case of
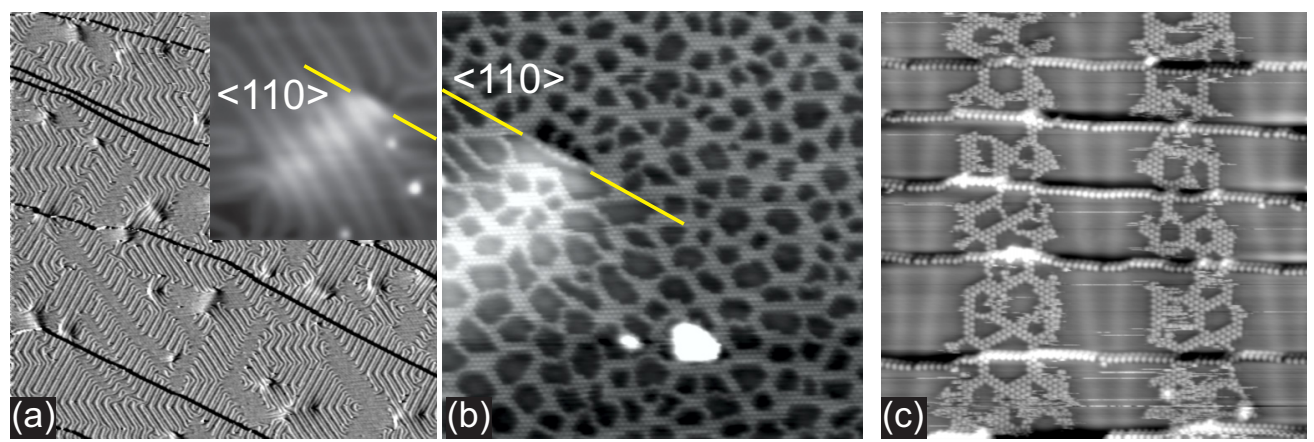

FIG. 8. (a) Clean $\mathrm{Au}(111)$ surface (derivative mode, $3000 \times 3000 \AA^{2}, \mathrm{U}_{s}=-100 \mathrm{mV}, \mathrm{I}_{t}=0.5 \mathrm{nA}, 5 \mathrm{~K}$ ) with numerous edge dislocations generated by subsurface argon bubbles. The inset shows STM image of the dislocation step segment aligned parallel to the $\langle 110\rangle$ direction. (b) Nanoporous superstructure $(\theta=0.13 \mathrm{ML})$ distorted by the presence of the edge dislocation $\left(372 \times 372 \AA^{2}, \mathrm{U}_{s}=-500 \mathrm{mV}, \mathrm{I}_{t}=0.75 \mathrm{nA}\right.$, $5 \mathrm{~K})$. The step segment decorated by chlorine atoms is visible as a straight line parallel to the $\langle 110\rangle$ direction. (c) Chlorine adsorbed on the vicinal $\mathrm{Au}(232321)$ surface $(\theta=0.06 \mathrm{ML})$ (image flattened, atomic steps are horizontal and fully decorated by chlorine atoms, $370 \times 370 \AA^{2}$, $\left.\mathrm{U}_{s}=-500 \mathrm{mV}, \mathrm{I}_{t}=0.75 \mathrm{nA}, 5 \mathrm{~K}\right)$. 
flat $\mathrm{Au}(111)]$, the structure of the chlorine layer on the vicinal surface appears to be different. According to the STM image, soliton lines do not disappear. Therefore, we can conclude that gold reconstruction is much preserved on the vicinal surface in comparison with the flat terraces. The possible explanation could be in an additional stress relaxation at the step edge that, in turn, can indicate the important role of elastic interactions in the $\mathrm{Cl} / \mathrm{Au}(111)$ system.

It is noteworthy that the formation of nanopatterns as a result of adsorption has also been reported for several systems: $\mathrm{Pb} / \mathrm{Cu}(111)$ [39,40], O/Cu(110) [36,41], N/Cu(100) [42,43]. In all these cases, the long-range elastic interactions also played a decisive role.

\section{CONCLUSIONS}

Thus, we have shown that chlorine dosing onto $\mathrm{Au}(111)$ at room temperature and subsequent cooling below $110 \mathrm{~K}$ results in the formation of specific atomic structures at low coverage. First, chlorine forms single-atomic chains in the fcc domains of the herringbone reconstruction. At coverage of $\approx 0.12$ $\mathrm{ML}$, the reconstruction disappears leaving on the surface a quasihexagonal superstructure consisting of nanopores with the average periodicity of $35 \AA$. At higher coverage, we detected a monotonic decrease of the average period of the nanoporous structure and its gradual conversion into the simple commensurate $(\sqrt{3} \times \sqrt{3}) R 30^{\circ}$ structure at $0.33 \mathrm{ML}$. On the basis of our experimental observations and DFT calculations, the driving force for the superstructure formation is attributed to long-range substrate-mediated elastic interactions.

\section{ACKNOWLEDGMENTS}

We acknowledge the financial support from The Russian Foundation for Basic Research (Grant No. 15-02-99542-a) and from the Program of Presidium of Russian Academy of Sciences No. 39 ("Physico-chemical problems of surface phenomena"). We are grateful to the Joint Supercomputer Center of RAS and to the Supercomputing Center of Lomonosov Moscow State University [44] for the possibility of using their computational resources for our calculations.
[1] N. D. Spencer and R. M. Lambert, Surf. Sci. 107, 237 (1981).

[2] G. N. Kastanas and B. E Koel, Appl. Surf. Sci. 64, 235 (1993).

[3] W. Gao, T. A. Baker, L. Zhou, D. S. Pinnaduwage, E. Kaxiras, and C. M. Friend, J. Am. Chem. Soc. 130, 3560 (2008).

[4] Z. V. Zheleva, V. R. Dhanak, and G. Held, Phys. Chem. Chem. Phys. 12, 10754 (2010)

[5] B. V. Andryushechkin, V. V. Cherkez, E. V. Gladchenko, T. V. Pavlova, G. M. Zhidomirov, B. Kierren, C. Didiot, Y. FagotRevurat, D. Malterre, and K. N. Eltsov, J. Phys. Chem. C 117, 24948 (2013).

[6] V. V. Zheltov, V. V. Cherkez, B. V. Andryushechkin, G. M. Zhidomirov, B. Kierren, Y. Fagot-Revurat, D. Malterre, and K. N. Eltsov, Phys. Rev. B 89, 195425 (2014).

[7] T. A. Baker, C. M. Friend, and E. Kaxiras, J. Am. Chem. Soc. 130, 3720 (2008).

[8] T. A. Baker, C. M. Friend, and E. Kaxiras, J. Chem. Phys. 129, 104702 (2008).

[9] I. Horcas, R. Fernandez, J. Gomez-Rodriguez, J. Colchero, J. Gomez-Herrero, and A. Baro, Rev. Sci. Instrum. 78, 013705 (2007).

[10] G. Kresse and J. Hafner, Phys. Rev. B 47, 558 (1993).

[11] G. Kresse and J. Hafner, Phys. Rev. B 49, 14251 (1994).

[12] G. Kresse and J. Furthmüller, Comput. Mater. Sci. 6, 15 (1996).

[13] G. Kresse and J. Furthmüller, Phys. Rev. B 54, 11169 (1996).

[14] J. P. Perdew, K. Burke, and M. Ernzerhof, Phys. Rev. Lett. 77, 3865 (1996).

[15] G. Kresse and D. Joubert, Phys. Rev. B 59, 1758 (1999).

[16] H. J. Monkhorst and J. D. Pack, Phys. Rev. B 13, 5188 (1976).

[17] J. V. Barth, H. Brune, G. Ertl, and R. J. Behm, Phys. Rev. B 42, 9307 (1990).

[18] S. Narasimhan and D. Vanderbilt, Phys. Rev. Lett. 69, 1564 (1992).

[19] D. D. Chambliss, R. J. Wilson, and S. Chiang, Phys. Rev. Lett. 66, 1721 (1991).

[20] B. Voigtländer, G. Meyer, and N. M. Amer, Phys. Rev. B 44, 10354 (1991).
[21] J. A. Meyer, I. D. Baikie, E. Kopatzki, and R. J. Behm, Surf. Sci 365, L647 (1996).

[22] F. Hanke and J. Björk, Phys. Rev. B 87, 235422 (2013).

[23] Y. Wang, N. S. Hush, and J. R. Reimers, Phys. Rev. B 75, 233416 (2007).

[24] C. E. Bach, M. Giesen, H. Ibach, and T. L. Einstein, Phys. Rev. Lett. 78, 4225 (1997).

[25] B. K. Min, A. R. Alemozafar, M. M. Biener, and C. M. Freind, Top. Catal. 36, 77 (2005).

[26] H. Walen, D.-J. Liu, J. Oh, H. Lim, J. W. Evans, Y. Kim, and P. A. Thiel, J. Chem. Phys. 143, 014704 (2015).

[27] A. D. Jewell, H. L. Tierney, and E. C. H. Sykes, Phys. Rev. B 82, 205401 (2010)

[28] J. V. Barth, R. J. Behm, and G. Ertl, Surf. Sci. 302, L319 (1994).

[29] A. Bogicevic, S. Ovesson, P. Hyldgaard, B. I. Lundqvist, H Brune, and D. R. Jennison, Phys. Rev. Lett. 85, 1910 (2000).

[30] K. A. Fichthorn and M. Scheffler, Phys. Rev. Lett. 84, 5371 (2000).

[31] M. L. Merrick, W. Luo, and K. A. Fichtorn, Prog. Surf. Sci. 72, 117 (2003).

[32] K. Lejaeghere, V. Van Speybroeck, G. Van Oost, and S. Cottenier, Crit. Rev. Solid State Mater. Sci. 39, 1 (2014).

[33] J. Engbæk, J. Schiøtz, B. Dahl-Madsen, and S. Horch, Phys. Rev. B 74, 195434 (2006).

[34] T. Michely and G. Comsa, J. Vac. Sci. Technol. B 9, 862 (1991).

[35] V. I. Marchenko, and A. Ya. Parshin, Zh. Eksp. Teor. Fiz. 79, 257 (1980) [Sov. Phys.-JETP 52, 129 (1980)].

[36] G. Prévot, B. Croset, Y. Girard, A. Coati, Y. Garreau, M. Hohage, L. D. Sun, and P. Zeppenfeld, Surf. Sci. 549, 52 (2004).

[37] G. Prévot and B. Croset, Phys. Rev. B 74, 235410 (2006).

[38] C. Didiot, S. Pons, B. Kierren, Y. Fagot-Revurat, and D. Malterre, Nat. Nanotechnol. 2, 617 (2007).

[39] R. Plass, J. A. Last, N. C. Bartelt, and G. L. Kellogg, Nature (London) 412, 875 (2001) 
[40] R. Plass, N. C. Bartelt, and G. L. Kellogg, J. Phys.: Condens. Matter 14, 4227 (2001).

[41] K. Kern, H. Niehus, A. Schatz, P. Zeppenfeld, J. Goerge, and G. Comsa, Phys. Rev. Lett. 67, 855 (1991).

[42] H. Ellmer, V. Repain, S. Rousset, B. Croset, M. Sotto, and P. Zeppenfeld, Surf. Sci. 476, 95 (2001).
[43] B. Croset, Y. Girard, G. Prévot, M. Sotto, Y. Garreau, R. Pinchaux, and M. Sauvage-Simkin, Phys. Rev. Lett. 88, 056103 (2002).

[44] V. Sadovnichy, A. Tikhonravov, Vl. Voevodin, and V. Opanasenko, in Contemporary High Performance Computing: From Petascale toward Exascale, edited by J. S. Vetter (CRC Press, Boca Raton, FL, 2013), pp. 283-307. 